\title{
Spherical Symmetric Solutions for the Motion of Relativistic Membranes in the Schwarzschild-Anti de Sitter Space-Time
}

\author{
Xiaoyan Li \\ Department of Mathematics, Zhejiang University, Hangzhou 310027, China \\ Correspondence should be addressed to Xiaoyan Li; lixiaoyanxl@126.com
}

Received 18 July 2013; Accepted 23 September 2013

Academic Editor: Laurent Gosse

Copyright (C) 2013 Xiaoyan Li. This is an open access article distributed under the Creative Commons Attribution License, which permits unrestricted use, distribution, and reproduction in any medium, provided the original work is properly cited.

This paper concerns motion of relativistic membranes in the Schwarzschild-anti de Sitter space-time. We derive a nonlinear equation for relativistic membranes moving in the Schwarzschild-anti de Sitter space-time, discuss spherical symmetric solutions for the motion equations, and obtain some interesting physical results.

\section{Introduction}

This paper concerns the motion of relativistic strings in the Schwarzschild-anti de Sitter space-time. The metric reads

$$
\begin{aligned}
d s^{2}= & -\left(1-\frac{2 m}{r}-\frac{\lambda}{3} r^{2}\right) d t^{2}+\left(1-\frac{2 m}{r}-\frac{\lambda}{3} r^{2}\right)^{-1} \\
& \times d r^{2}+r^{2}\left(d \theta^{2}+\sin ^{2} \theta d \varphi^{2}\right),
\end{aligned}
$$

which is a spherically symmetric solution of the Einstein field equations. The Schwarzschild-anti de Sitter space-time is a fundamental physical space-time; it plays an important role in general relativity, the theory of black holes, and modern cosmology.

As is well known, the theory of minimal surfaces/submanifolds has a long history originating from the papers of Lagrange in 1760 and the famous Plateau problem. And this theory plays a significant role in general relativity, the theory of black holes, particle physics, and so on. A good deal of attention has been paid to the theory of minimal surfaces in the Euclidean space $\mathbb{R}^{n}$ and Riemannian manifolds in recent years. On this topic, we refer to two classical books $[1,2]$. For the theory of extremal surfaces/submanifolds in the Minkowski space-time, many important results have been derived ([3-6]). Particularly, the theory of extremal surfaces/submanifolds is very important in elementary particle physics. It is a relativistic string model that deals with a onedimensional relativistic object, whose world surface is an extremal surface in the Minkowski space-time [7]. For the relativistic string theory, we refer to an excellent book by Barbashov and Nesterenko [8].

The authors in [9] simplified the description of relativistic membrane in the Minkowski space-time by the lightcone gauge. By variables transformations, a relationship was established between the dynamics of relativistic membrane and two-dimensional fluid dynamics. Moreover, in [10] they obtained a vector-valued equation of first order for relativistic membrane by introducing the orthonormal $(1+3)$-gauge, and then they deduced a second-order equation for minimal graph $z=z\left(t, x_{1}, x_{2}\right)$ in the Minkowski space-time $\mathbb{R}^{1+3}$ by the hodograph technique. Furthermore, using reduction of membrane equation in light-cone gauge, they obtained a second-order partial differential equation for the velocity potential. In the paper [11], a lot of simplifications of the equations of motion for the relativistic membrane were exhibited such as the orthonormal light-cone gauge, minimal graph method, and level set method. According to these reformulations, Hoppe found some classical solutions for the equations governing the motion of relativistic membrane in the Minkowski space-time.

Here we want to mention a result in [12]: the authors investigated the basic equations for the motion of relativistic membranes in the Schwarzschild space-time and got a nonlinear wave equation, and then they studied a spherical symmetric solution for the motion of relativistic membranes, giving many new physical results. By variational 
and geometrical methods, Huang and Kong in [13] obtained a new kind of equation describing the motion of relativistic membranes in the Minkowski space-time $\mathbb{R}^{1+n}(n \geqslant 3)$, gave some interesting properties, and showed that all planewave solutions of these equations were light-like extremal submanifolds and vice versa except for a type of special solution.

Kong and Zhang studied the motion of relativistic closed strings in the Minkowski space $\mathbb{R}^{1+n}$ in [14]; particularly, the authors obtained a general solution formula for this system of nonlinear equations. Based on the solution formula, they showed that the motion of closed strings was always time-periodic and extended the solution formula to finite relativistic strings. Moreover, in [15], Kong et al. investigated the dynamics of relativistic strings in the Minkowski spacetime $\mathbb{R}^{1+n}(n \geq 2)$. They first obtained a system with $n$ nonlinear wave equations of Born-Infeld type describing the motion of the string, and then they showed that this system enjoyed some interesting geometric properties; in the end, they gave a sufficient and necessary condition for the global existence of extremal surfaces without a space-like point in $\mathbb{R}^{1+n}$. Furthermore, they made a lot of numerical analyses demonstrating that various topological singularities developed in finite time in the motion of the string.

This paper mainly focuses on the equations and spherical symmetric solutions for the motion of relativistic membranes in the Schwarzschild-anti de Sitter space-time. Concretely, we derive an interesting nonlinear wave equation for relativistic membranes and study systematically the spherical symmetric solutions for the motion of membranes.

The paper is organized as follows. In Section 2, we recall the basic equations for the motion of relativistic membranes in the Schwarzschild-anti de Sitter space-time. Section 3 is devoted to a systematical study on the spherical symmetric solutions of the equations for the motion of relativistic membranes; at the same time, some new and interesting physical phenomena are discovered and illustrated. Some discussions are given in Section 4.

\section{Basic Equation}

A four-dimensional Lorentzian manifold $\left(M, g_{\mu \nu}\right)$ is called the Schwarzschild-anti de Sitter space-time if the metric of $M$ can be written as (2) with $\lambda<0$ ([16]). Consider

$$
\begin{aligned}
d s^{2}= & g_{\mu \nu} d x^{\mu} d x^{\nu} \\
= & -\left(1-\frac{2 m}{r}-\frac{\lambda}{3} r^{2}\right) d t^{2} \\
& +\left(1-\frac{2 m}{r}-\frac{\lambda}{3} r^{2}\right)^{-1} \\
& \times d r^{2}+r^{2}\left(d \theta^{2}+\sin ^{2} \theta d \varphi^{2}\right),
\end{aligned}
$$

where $m$ is a positive constant representing the mass of the universe and $\lambda$ is the cosmological constant. Assume $X=$ $(t, r, \theta, \varphi)$ is a position vector of a point in the Schwarzschildanti de Sitter space-time. Moreover, let $r_{+}$be the largest root of the equation $1-2 m / r-(\lambda / 3) r^{2}=0$. Obviously, it holds that $0<r_{+}<2 m$. Since we are only interested in the motion of membrane in the region $r>r_{+}$, we may suppose that $r_{0}>r_{+}$.

Consider the motion of a relativistic membrane in the Schwarzschild-anti de Sitter space-time

$$
(t, \theta, \varphi) \longrightarrow(t, r(t, \theta, \varphi), \theta, \varphi)
$$

In the coordinates $(t, \theta, \varphi)$, the induced metric of the submanifold $\mathscr{M}$ is

$$
d s^{2}=(d t, d \theta, d \varphi) \mathscr{G}(d t, d \theta, d \varphi)^{T}
$$

where

$$
\mathscr{G}=\left(\begin{array}{lll}
g_{00} & g_{01} & g_{02} \\
g_{10} & g_{11} & g_{12} \\
g_{20} & g_{21} & g_{22}
\end{array}\right),
$$

in which

$$
\begin{aligned}
& g_{00}=-\left(1-\frac{2 m}{r}-\frac{\lambda}{3} r^{2}\right)+\left(1-\frac{2 m}{r}-\frac{\lambda}{3} r^{2}\right)^{-1} r_{t}^{2}, \\
& g_{01}=g_{10}=\left(1-\frac{2 m}{r}-\frac{\lambda}{3} r^{2}\right)^{-1} r_{t} r_{\theta}, \\
& g_{02}=g_{20}=\left(1-\frac{2 m}{r}-\frac{\lambda}{3} r^{2}\right)^{-1} r_{t} r_{\varphi}, \\
& g_{11}=r^{2}+\left(1-\frac{2 m}{r}-\frac{\lambda}{3} r^{2}\right)^{-1} r_{\theta}^{2}, \\
& g_{12}=g_{21}=\left(1-\frac{2 m}{r}-\frac{\lambda}{3} r^{2}\right)^{-1} r_{\theta} r_{\varphi}, \\
& g_{22}=r^{2} \sin ^{2} \theta+\left(1-\frac{2 m}{r}-\frac{\lambda}{3} r^{2}\right)^{-1} r_{\varphi}^{2} .
\end{aligned}
$$
that is,

We assume that the submanifold $\mathscr{M}$ is $C^{2}$ and time-like;

$$
\begin{aligned}
\Delta \triangleq & \operatorname{det} \mathscr{G}=-\left(1-\frac{2 m}{r}-\frac{\lambda}{3} r^{2}\right) r^{4} \sin ^{2} \theta-r^{2} r_{\varphi}^{2} \\
& -r^{2} \sin ^{2} \theta r_{\theta}^{2}+\left(1-\frac{2 m}{r}-\frac{\lambda}{3} r^{2}\right)^{-1} \\
& \times r^{4} \sin ^{2} \theta r_{t}^{2}<0 .
\end{aligned}
$$

The area element of $\mathscr{M}$ is

$$
d \mathscr{A}=\sqrt{-\Delta} d t d \theta d \varphi .
$$

And the submanifold $\mathscr{M}$ is called extremal if $r=r(t, \theta, \varphi)$ is a critical point of the area functional

$$
\mathscr{I}=\iiint \sqrt{-\Delta} d t d \theta d \varphi
$$




$$
\begin{array}{ll}
\begin{array}{ll}
\text { By calculations, we obtain the corresponding Euler- } \\
\text { Lagrange equation as follows: }
\end{array} & -\frac{r^{4} r_{t} \sin ^{2} \theta}{2 \Delta}\left(1-\frac{2 m}{r}-\frac{\lambda}{3} r^{2}\right)^{-1} \\
\begin{array}{cc}
{\left[\left(1-\frac{2 m}{r}-\frac{\lambda}{3} r^{2}\right)^{-1}-\frac{1}{\Delta}\left(1-\frac{2 m}{r}-\frac{\lambda}{3} r^{2}\right)^{-2} r^{4} \sin ^{2} \theta r_{t}^{2}\right]} & \times\left[-2 r r_{t} r_{\theta}^{2} \sin ^{2} \theta+r^{3} r_{t} \sin ^{2} \theta\right. \\
\times r^{4} \sin ^{2} \theta r_{t t}-\left[r^{2}+\frac{1}{\Delta} r^{4} r_{\varphi}^{2}\right] r_{\varphi \varphi} & \times\left(-4+\frac{6 m}{r}+2 \lambda r^{2}\right)+r^{3} r_{t}^{3} \sin ^{2} \theta \\
-\left[1+\frac{1}{\Delta} r^{2} \sin ^{2} \theta r_{\theta}^{2}\right] r^{2} \sin ^{2} \theta r_{\theta \theta}+\frac{2}{\Delta} & \times\left(1-\frac{2 m}{r}-\frac{\lambda}{3} r^{2}\right)^{-2} \\
\times\left(1-\frac{2 m}{r}-\frac{\lambda}{3} r^{2}\right)^{-1} r^{6} \sin ^{2} \theta r_{t} r_{\varphi} r_{t \varphi} & \left.\times\left(4-\frac{10 m}{r}-\frac{2 \lambda}{3} r^{2}\right)-2 r r_{t} r_{\varphi}^{2}\right]=0 .
\end{array}
\end{array}
$$$$
-\frac{2}{\Delta} r^{4} \sin ^{2} \theta r_{\theta} r_{\varphi} r_{\theta \varphi}
$$$$
+\frac{2}{\Delta}\left(1-\frac{2 m}{r}-\frac{\lambda}{3} r^{2}\right)^{-1} r^{6} \sin ^{4} \theta r_{t} r_{\theta} r_{t \theta}
$$$$
+r^{3} \sin ^{2} \theta\left(2-\frac{3 m}{r}-\lambda r^{2}\right)-r r_{\varphi}^{2}
$$$$
-r r_{\theta}^{2} \sin ^{2} \theta-r^{2} r_{\theta} \sin 2 \theta
$$$$
+r_{t}^{2} r^{3} \sin ^{2} \theta\left(1-\frac{2 m}{r}-\frac{\lambda}{3} r^{2}\right)^{-2}
$$$$
\times\left(2-\frac{5 m}{r}-\frac{\lambda}{3} r^{2}\right)+\frac{r^{2} r_{\varphi}}{2 \Delta}
$$$$
\times\left[-2 r r_{\varphi} r_{\theta}^{2} \sin ^{2} \theta\right.
$$$$
+\left(-4+\frac{6 m}{r}+2 \lambda r^{2}\right)
$$$$
\times r^{3} \sin ^{2} \theta r_{\varphi}+\left(1-\frac{2 m}{r}-\frac{\lambda}{3} r^{2}\right)^{-2}
$$$$
\times r_{t}^{2} r^{3} \sin ^{2} \theta r_{\varphi}\left(4-\frac{10 m}{r}-\frac{2 \lambda}{3} r^{2}\right)
$$$$
\left.-2 r r_{\varphi}^{3}\right]+\frac{r^{2} r_{\theta} \sin ^{2} \theta}{2 \Delta}
$$$$
\times\left[-\left(1-\frac{2 m}{r}-\frac{\lambda}{3} r^{2}\right) r^{4} \sin 2 \theta\right.
$$$$
-2 r r_{\theta} r_{\varphi}^{2}-2 r r_{\theta}^{3} \sin ^{2} \theta-r^{2} r_{\theta}^{2} \sin 2 \theta
$$$$
+\left(1-\frac{2 m}{r}-\frac{\lambda}{3} r^{2}\right)^{-1} r_{t}^{2} r^{4} \sin 2 \theta
$$$$
+r^{3} r_{\theta} \sin ^{2} \theta r_{t}^{2}\left(1-\frac{2 m}{r}-\frac{\lambda}{3} r^{2}\right)^{-2}
$$$$
\times\left(4-\frac{10 m}{r}-\frac{2 \lambda}{3} r^{2}\right)
$$$$
\left.+r^{3} r_{\theta} \sin ^{2} \theta\left(-4+\frac{6 m}{r}+2 \lambda r^{2}\right)\right]
$$

\section{Spherical Symmetric Solutions}

This section is concentrated on spherical symmetric solutions for the motion of relativistic membranes. Now we study the spherical symmetric solutions $r=r(t)$ for the motion of relativistic membranes. Therefore, in the present situation, (10) can be simplified as the following ordinary differential equation:

$$
\begin{aligned}
r_{t t}= & \frac{2 r-m-(5 / 3) \lambda r^{3}}{r\left(r-2 m-(\lambda / 3) r^{3}\right)} r_{t}^{2} \\
& -\frac{\left(r-2 m-(\lambda / 3) r^{3}\right)\left(2 r-3 m-\lambda r^{3}\right)}{r^{3}} .
\end{aligned}
$$
data

Consider the initial value problem for (11) with the initial

$$
r(0)=r_{0}, \quad r_{t}(0)=r_{1} \text {, }
$$

where $r_{0}$ and $r_{1}$ are two constants meaning the initial position and initial velocity of the membrane, respectively. Since we are only interested in the motion of the membrane in the region $r>r_{+}$, without loss of generality, we may assume that $r_{0}>r_{+}$.

Let

$$
z=r_{t}
$$

Equation (11) becomes the following form:

$$
\begin{aligned}
\frac{d z}{d r}= & \frac{2 r-m-(5 / 3) \lambda r^{3}}{r\left(r-2 m-(\lambda / 3) r^{3}\right)} z \\
& -\frac{\left(r-2 m-(\lambda / 3) r^{3}\right)\left(2 r-3 m-\lambda r^{3}\right)}{r^{3}} \frac{1}{z} .
\end{aligned}
$$


Using (14), we derive

$$
\begin{aligned}
\frac{d}{d r}( & \left.\frac{z^{2}}{r\left(r-2 m-(\lambda / 3) r^{3}\right)^{3}}\right) \\
= & \frac{d}{d r}\left(\frac{1}{r\left(r-2 m-(\lambda / 3) r^{3}\right)^{3}}\right) z^{2} \\
& +\frac{2 z}{r\left(r-2 m-(\lambda / 3) r^{3}\right)^{3}} \frac{d z}{d r} \\
= & -\frac{2\left(2 r-3 m-\lambda r^{3}\right)}{r^{4}\left(r-2 m-(\lambda / 3) r^{3}\right)^{2}} \\
= & \frac{d}{d r}\left(\frac{1}{r^{3}\left(r-2 m-(\lambda / 3) r^{3}\right)}\right) .
\end{aligned}
$$

Integrating (15), we have

$$
\begin{aligned}
z^{2}= & \frac{\left(r-2 m-(\lambda / 3) r^{3}\right)^{2}}{r^{2}} \\
& \times\left[1+F\left(r_{0}, r_{1}\right) r^{3}\left(r-2 m-\frac{\lambda}{3} r^{3}\right)\right],
\end{aligned}
$$

where

$$
\begin{aligned}
F\left(r_{0}, r_{1}\right)= & \frac{r_{1}^{2}}{r_{0}\left(r_{0}-2 m-(\lambda / 3) r_{0}^{3}\right)^{3}} \\
& -\frac{1}{r_{0}^{3}\left(r_{0}-2 m-(\lambda / 3) r_{0}^{3}\right)} .
\end{aligned}
$$

Noting (16), we obtain from (11) that

$$
\begin{aligned}
r_{t t}=\frac{r-2 m-(\lambda / 3) r^{3}}{r^{3}}[ & 2 m-\frac{2}{3} \lambda r^{3} \\
& +F\left(r_{0}, r_{1}\right) r^{3}\left(r-2 m-\frac{\lambda}{3} r^{3}\right) \\
& \left.\times\left(2 r-m-\frac{5}{3} \lambda r^{3}\right)\right] .
\end{aligned}
$$

Define two functions $f(r)$ and $g(r)$ as follows:

$$
\begin{gathered}
f(r)=r^{3}\left(r-2 m-\frac{\lambda}{3} r^{3}\right) \\
g(r)=r^{3}\left(r-2 m-\frac{\lambda}{3} r^{3}\right)\left(2 r-m-\frac{5}{3} \lambda r^{3}\right) .
\end{gathered}
$$

Obviously $f(r)$ and $g(r)$ are strictly increasing positive functions when $r>r_{+}$. Hence, using the notations $f(r)$ and $g(r),(16)$ and (18) are written as

$$
\begin{aligned}
z^{2} & =\frac{\left(r-2 m-(\lambda / 3) r^{3}\right)^{2}}{r^{2}}\left[1+F\left(r_{0}, r_{1}\right) f(r)\right], \\
r_{t t} & =\frac{r-2 m-(\lambda / 3) r^{3}}{r^{3}}\left[2 m-\frac{2}{3} \lambda r^{3}+F\left(r_{0}, r_{1}\right) g(r)\right],
\end{aligned}
$$

respectively.
In order to solve the Cauchy problem (11) and (12), that is, (21) and (12), we distinguish several cases as follows.

Case 1. Consider

$$
r_{1} \geqslant \frac{r_{0}-2 m-(\lambda / 3) r_{0}^{3}}{r_{0}} .
$$

In this case, noting $r_{0}>r_{+}$, it holds that

$$
F\left(r_{0}, r_{1}\right) \geqslant 0, \quad r_{t}(0)=r_{1}>0, \quad r_{t t}(0)>0 .
$$

According to the properties of $f(r)$ and $g(r)$, it follows from (20) that

$$
z=r_{t}>0
$$

which implies that $r$ is a strictly increasing function of $t$. Therefore, it follows that $r(t)>r(0)=r_{0}>r_{+}$for $t>0$. Thus, we obtain from (21) that

$$
r_{t t}>0
$$

Case 1(a) $r_{1}=\left(r_{0}-2 m-(\lambda / 3) r_{0}^{3}\right) / r_{0}$.

In the present situation, we have $F\left(r_{0}, r_{1}\right)=0$, and

$$
\begin{gathered}
r_{t}=\frac{r-2 m-(\lambda / 3) r^{3}}{r} \\
r_{t t}=\frac{r-2 m-(\lambda / 3) r^{3}}{r^{3}}\left(2 m-\frac{2}{3} \lambda r^{3}\right) .
\end{gathered}
$$

Clearly, because $r_{t}>0$ and $r_{t t}>0, r(t)$ tends to the infinity. We next prove that $r(t)$ goes to the positive infinity in finite time. In fact, it follows from (26) that

$$
t=\int_{r_{0}}^{r} \frac{r}{r-2 m-(\lambda / 3) r^{3}} d r
$$

Let

$$
T_{\max }=\int_{r_{0}}^{\infty} \frac{r}{r-2 m-(\lambda / 3) r^{3}} d r .
$$

It is easy to see that $t \leq T_{\max }$. Now we show that $T_{\max }<\infty$. For a certain large $\widetilde{r}$,

$$
\begin{aligned}
T_{\max } & =\int_{r_{0}}^{\tilde{r}} \frac{r}{r-2 m-(\lambda / 3) r^{3}} d r+\int_{\tilde{r}}^{\infty} \frac{r}{r-2 m-(\lambda / 3) r^{3}} d r \\
& <\int_{r_{0}}^{\tilde{r}} \frac{r}{r-2 m-(\lambda / 3) r^{3}} d r+\int_{\widetilde{r}}^{\infty} \frac{r}{-(\lambda / 3) r^{3}} d r \\
& =\int_{r_{0}}^{\tilde{r}} \frac{r}{r-2 m-(\lambda / 3) r^{3}} d r-\frac{3}{\lambda \widetilde{r}} \\
& <\infty,
\end{aligned}
$$

which implies

$$
r(t) \longrightarrow+\infty, \quad \text { when } t \nearrow T_{\max } .
$$



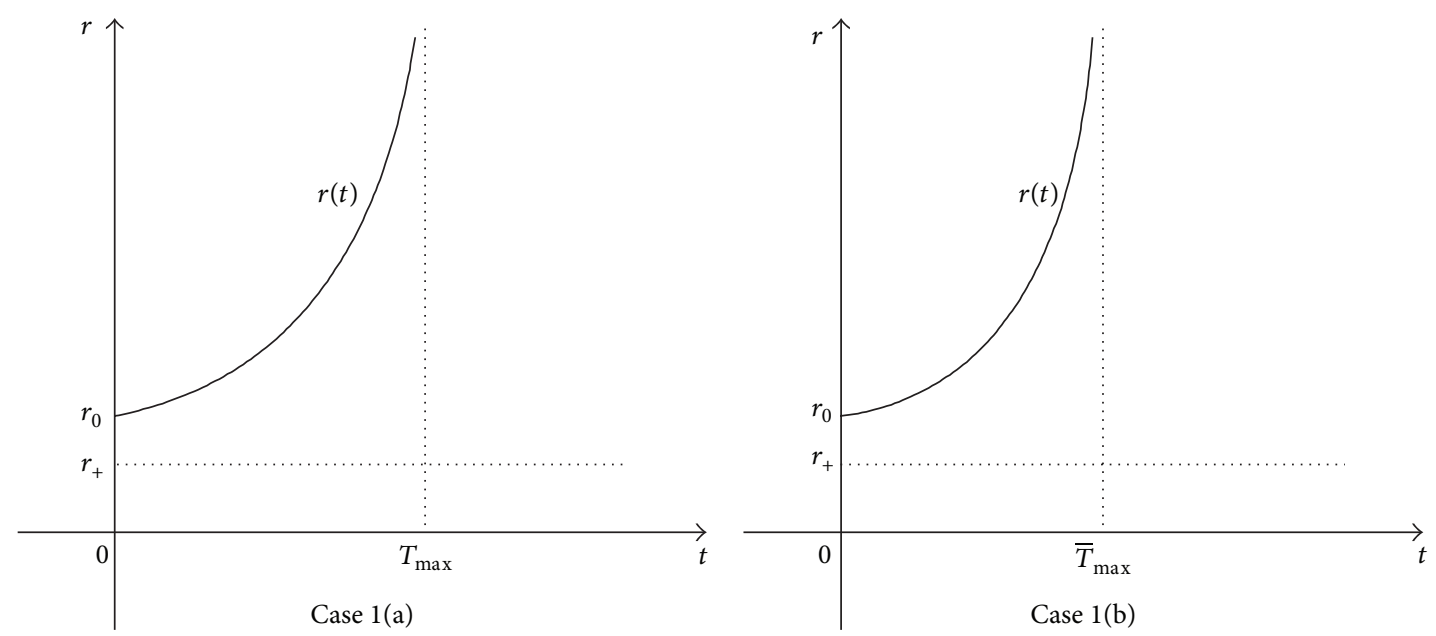

FIGURE 1: The graph of the solution $r=r(t)$ for Cases 1(a) and 2(b).

Equation (30) shows that the solution of the initial value problem (11) and (12) must blow up in finite time and the life span is $\left[0, T_{\max }\right.$ ), where $T_{\max }$ is defined by (28). Different from the case that initial velocity is equal to critical velocity in [12], where the solution $r=r(t)$ of the Cauchy problem exists globally on the time $t \in[0, \infty)$, in this paper the solution of the initial value problem (11) and (12) blows up in finite time when initial velocity is equal to critical velocity. See Figure 1 for the graph of the solution $r=r(t)$ in the present situation.

Case $1(\mathrm{~b}) r_{1}>\left(r_{0}-2 m-(\lambda / 3) r_{0}^{3}\right) / r_{0}$.

In this case, we have $F\left(r_{0}, r_{1}\right)>0$. Since $r_{t}>0$ and $r_{t t}>$ $0, r(t)$ goes to the infinity. In what follows, we prove that $r(t)$ tends to the positive infinity in finite time.

Similar to the above case, it follows from (16) that

$$
\begin{aligned}
t=\int_{r_{0}}^{r} & \frac{r}{r-2 m-(\lambda / 3) r^{3}} \\
& \times \frac{1}{\sqrt{1+F\left(r_{0}, r_{1}\right) r^{3}\left(r-2 m-(\lambda / 3) r^{3}\right)}} d r .
\end{aligned}
$$

Denote

$$
\begin{aligned}
\bar{T}_{\max }=\int_{r_{0}}^{\infty} \frac{r}{r-2 m-(\lambda / 3) r^{3}} \\
\quad \times \frac{1}{\sqrt{1+F\left(r_{0}, r_{1}\right) r^{3}\left(r-2 m-(\lambda / 3) r^{3}\right)}} d r .
\end{aligned}
$$

Obviously, we have $t \leqslant \bar{T}_{\max }$.
We next show that $\bar{T}_{\max }<\infty$. For a certain large $\bar{r}$,

$$
\begin{aligned}
\bar{T}_{\max }= & \int_{r_{0}}^{\bar{r}} \frac{r}{r-2 m-(\lambda / 3) r^{3}} \\
& \times \frac{1}{\sqrt{1+F\left(r_{0}, r_{1}\right) r^{3}\left(r-2 m-(\lambda / 3) r^{3}\right)}} d r \\
& +\int_{\bar{r}}^{\infty} \frac{r}{r-2 m-(\lambda / 3) r^{3}} \\
< & \times \frac{1}{\sqrt{1+F\left(r_{0}, r_{1}\right) r^{3}\left(r-2 m-(\lambda / 3) r^{3}\right)}} d r \\
& +\int_{r_{0}}^{\bar{r}} \frac{r}{r-2 m-(\lambda / 3) r^{3}} \\
= & \int_{r_{0}}^{\bar{r}} \frac{\frac{r}{r-2 m-(\lambda / 3) r^{3}}}{\sqrt{1+F\left(r_{0}, r_{1}\right) r^{3}\left(r-2 m-(\lambda / 3) r^{3}\right)}} d r \\
& \times \frac{r / 3) r^{6} \sqrt{-(\lambda / 3) F\left(r_{0}, r_{1}\right)}}{\sqrt{1+F\left(r_{0}, r_{1}\right) r^{3}\left(r-2 m-(\lambda / 3) r^{3}\right)}} d r \\
& +(4 / 3) \lambda \sqrt{-(\lambda / 3) F\left(r_{0}, r_{1}\right) \bar{r}^{4}} \\
& \times \infty .
\end{aligned}
$$

This proves that

$$
r(t) \longrightarrow+\infty, \quad \text { when } t \nearrow \bar{T}_{\max } .
$$


Equation (34) implies that the solution of the Cauchy problem (11)-(12) must blow up in finite time and the life span is the interval $\left[0, \bar{T}_{\max }\right)$, where $\bar{T}_{\max }$ is defined by (32). Compared to the case that initial velocity is larger than critical velocity in [12], in the present paper $\bar{T}_{\max }$ is controlled by the constant $\lambda$. See Figure 1 for the graph of the solution $r=r(t)$.

Case 2. Consider

$$
\begin{gathered}
\frac{r_{0}-2 m-(\lambda / 3) r_{0}^{3}}{r_{0}} \sqrt{\frac{2 r_{0}-3 m-\lambda r_{0}^{3}}{2 r_{0}-m-(5 / 3) \lambda r_{0}^{3}}} \\
<r_{1}<\frac{r_{0}-2 m-(\lambda / 3) r_{0}^{3}}{r_{0}} .
\end{gathered}
$$

In this situation, we obtain from (17) and (21) that

$$
\begin{gathered}
-\frac{2 m-(2 / 3) \lambda r_{0}^{3}}{g\left(r_{0}\right)}<F\left(r_{0}, r_{1}\right)<0, \\
r_{t}(0)=r_{1}>0, \quad r_{t t}(0)>0 .
\end{gathered}
$$

By the properties of $f(r)$ and $g(r)$, there exists a point $r^{*} \epsilon$ $\left(r_{+},+\infty\right)$ such that

$$
f\left(r^{*}\right)=-\frac{1}{F\left(r_{0}, r_{1}\right)} .
$$

Thus, it follows from (20) that

$$
z=r_{t}=0 \text {, when } r=r^{*} \text {. }
$$

On the one hand, noting (20) and the second inequality in (36), $r(t)$ will increase until a time $t^{*}>0$. At the time $t^{*}$, it holds that

$$
r_{t t}<0 \text {, when } r=r^{*} \text {. }
$$

By (36), we have $r_{t t}>0$, when $r=r_{0}$. So there exists a point $r_{\star} \in\left(r_{0}, r^{*}\right)$, such that

$$
r_{t t}=0, \quad \text { when } r=r_{\star} .
$$

On the other hand, when $r \rightarrow r_{+}+0$,

$$
r_{t} \longrightarrow 0, \quad r_{t t} \longrightarrow 0
$$

For $r \rightarrow r_{+}+\delta$ ( $\delta$ is sufficiently small and positive), both $r-2 m-(\lambda / 3) r^{3}$ and $g(r)$ are small enough and positive; thus $r_{t t}$ is sufficiently small and $r_{t t}>0$. Combined with (39), it holds that there exists a point $r_{*} \in\left(r_{+}+\delta, r^{*}\right)$ such that

$$
r_{t t}=0 \text {, when } r=r_{*} \text {. }
$$

Now we will discuss the developing with time of the membrane, that is, the properties enjoyed by the solution of the Cauchy problem (11) and (12). At the time $t^{*}$, it holds that

$$
r=r^{*}, \quad r_{t}=0, \quad r_{t t}<0 .
$$

Therefore, $r_{t}(t)<0$ for $t>t^{*}$. That is, when $t>t^{*}, r(t)$ is a strictly decreasing function; then there exists a time $t_{*}$ such that

$$
r\left(t_{*}\right)=r_{*}, \quad r_{t}\left(t_{*}\right)<0, \quad r_{t t}\left(t_{*}\right)=0 .
$$

Hence, by (20) and (21), we obtain that for $t>t_{*}$

$$
r(t)<r_{*}, \quad r_{t}(t)<0, \quad r_{t t}(t)>0 .
$$

Furthermore, from the fact that (42) holds when $r \rightarrow r_{+}+$ 0 , we see that the solution of the Cauchy problem (11) and (12) exists globally on the time $t \in[0, \infty)$, and the solution satisfies the following decay properties:

$$
r(t) \longrightarrow r_{+}, \quad r_{t}(t) \longrightarrow 0, \quad r_{t t}(t) \longrightarrow 0,
$$

when $t \rightarrow \infty$. Figure 2 is the graph of the solution for Case 2.

Case 3. Consider

$$
\begin{aligned}
0 & <r_{1} \leqslant \frac{r_{0}-2 m-(\lambda / 3) r_{0}^{3}}{r_{0}} \\
& \times \sqrt{\frac{2 r_{0}-3 m-\lambda r_{0}^{3}}{2 r_{0}-m-(5 / 3) \lambda r_{0}^{3}}} .
\end{aligned}
$$

In this situation, it follows from (17) and (21) that

$$
\begin{gathered}
-\frac{1}{f\left(r_{0}\right)}<F\left(r_{0}, r_{1}\right) \leqslant-\frac{2 m-(2 / 3) \lambda r_{0}^{3}}{g\left(r_{0}\right)}, \\
r_{t}(0)=r_{1}>0, \quad r_{t t}(0) \leqslant 0 .
\end{gathered}
$$

Similar to Case 2, we can exactly analyze the properties enjoyed by the solution of the Cauchy problem (11) and (12). The graph of the solution is shown in Figure 2. Figure 2 shows that, in this case, the solution of the Cauchy problem (11) and (12) exists globally on the time $t \in[0, \infty)$, and the solution satisfies the decay condition (46).

Case 4. Consider

$$
\begin{aligned}
& -\frac{r_{0}-2 m-(\lambda / 3) r_{0}^{3}}{r_{0}} \\
& \quad \times \sqrt{\frac{2 r_{0}-3 m-\lambda r_{0}^{3}}{2 r_{0}-m-(5 / 3) \lambda r_{0}^{3}}}<r_{1} \leqslant 0 .
\end{aligned}
$$

In this case, it holds that

$$
\begin{gathered}
-\frac{1}{f\left(r_{0}\right)} \leq F\left(r_{0}, r_{1}\right)<-\frac{2 m-(2 / 3) \lambda r_{0}^{3}}{g\left(r_{0}\right)}, \\
r_{t}(0)=r_{1} \leqslant 0, \quad r_{t t}(0)<0 .
\end{gathered}
$$

Similar to the discussion in Cases 2 and 3, we can exactly analyze the properties enjoyed by the solution of the Cauchy problem (11) and (12). See Case 4 in Figure 3 for the graph of the solution. In this case, the solution of the Cauchy problem (11) and (12) also exists globally on the time $t \in[0, \infty)$, and the solution $r=r(t)$ satisfies the decay condition (46). 

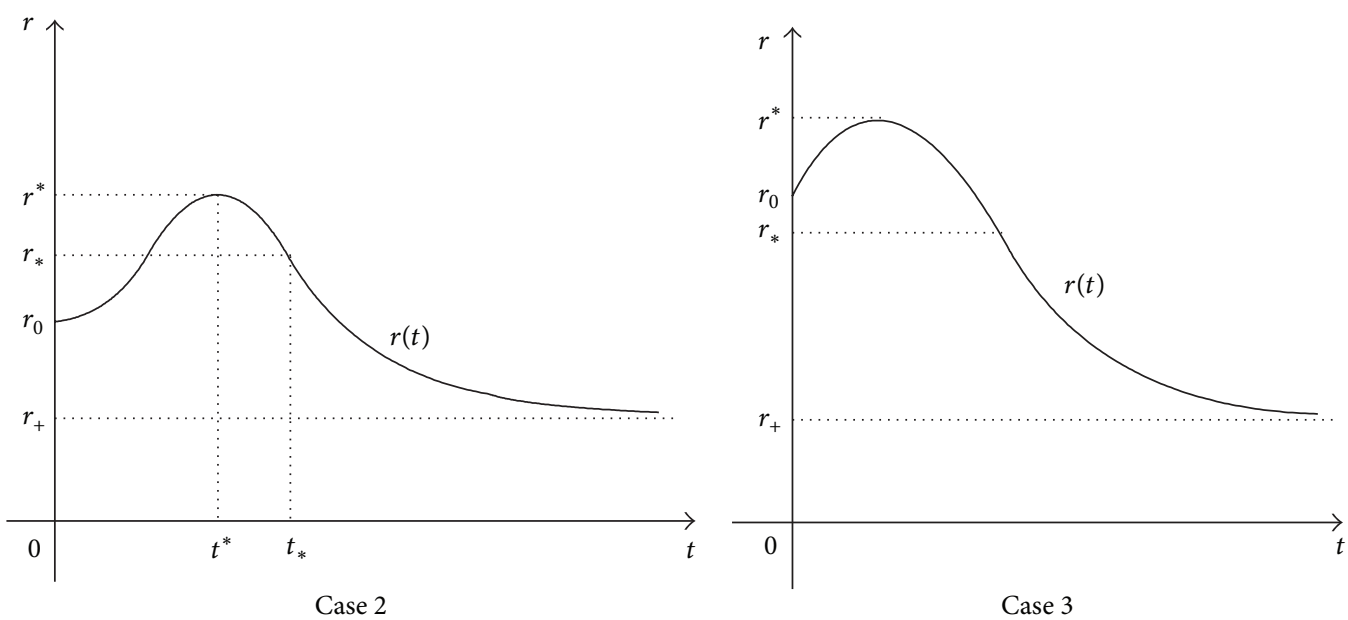

FIgURE 2: The graph of the solution $r=r(t)$ for Cases 2 and 3.
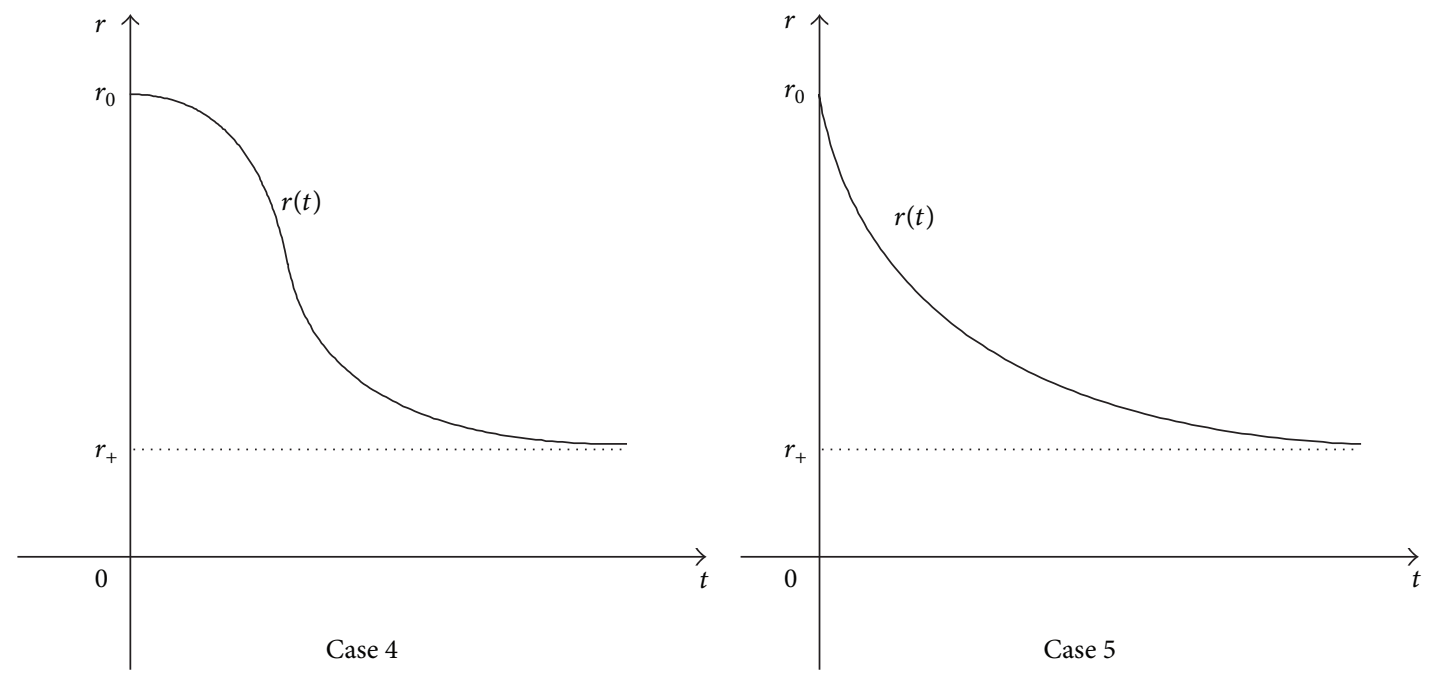

Figure 3: The graph of the solution $r=r(t)$ for Cases 4 and 5 .

Case 5. Consider

$$
\begin{aligned}
r_{1} \leqslant & -\frac{r_{0}-2 m-(\lambda / 3) r_{0}^{3}}{r_{0}} \\
& \times \sqrt{\frac{2 r_{0}-3 m-\lambda r_{0}^{3}}{2 r_{0}-m-(5 / 3) \lambda r_{0}^{3}}} .
\end{aligned}
$$

In this case, we have

$$
\begin{aligned}
& F\left(r_{0}, r_{1}\right) \geqslant-\frac{2 m-(2 / 3) \lambda r_{0}^{3}}{g\left(r_{0}\right)}, \\
& r_{t}(0)=r_{1}<0, \quad r_{t t}(0) \geqslant 0 .
\end{aligned}
$$

In the present situation we only consider the case that $F\left(r_{0}, r_{1}\right) \geqslant 0$; then it follows from (21) that

$$
r_{t t}>0
$$

provided that $r>r_{+}$.
Therefore, noting (53) and using (42), we know that the solution of the Cauchy problem (11) and (12) exists globally on the time $t \in[0, \infty)$, and the solution $r=r(t)$ satisfies the decay conditions (46). The graph and detailed information of the solution in the present situation are shown in Figure 3.

\section{Discussions}

The Schwarzschild-anti de Sitter space-time is a fundamental physical space-time and plays an important role in general relativity and the theory of black holes. The notion of extremal surfaces/submanifolds is used to formulate the Wilson criterion of quark confinement in gauge models of strong interactions. The theory of extremal surfaces/submanifolds is important in the elementary particle physics. The world sheets of relativistic membranes moving in physical space-times are nothing but extremal surfaces/submanifolds in these spacetimes. Some beautiful and deep results have been obtained, but unfortunately only a few results have been known for 
relativistic membranes moving in the Schwarzschild-anti de Sitter space-time.

In the present paper, we study the equations and spherical symmetric solutions for relativistic membranes moving in the Schwarzschild-anti de Sitter space-time. First, a nonlinear wave equation for the motion of relativistic membranes in the Schwarzschild-anti de Sitter space-time is derived, and then spherical symmetric solutions for the motion of relativistic membranes are studied systematically. We can summarize the main results as follows. In this paper we consider a spherical membrane centered at the black hole $r=0$ and assume that $r_{0}$ and $r_{1}$ represent the initial position and initial velocity of the membrane, respectively. For a given initial position $r_{0}>r_{+}$, we find a critical initial velocity $v_{c}=\left(r_{0}-2 m-(\lambda / 3) r_{0}^{3}\right) / r_{0}$ such that the motion with a different initial velocity has essentially different behavior. Exactly, (i) when $r_{1}>v_{c}$, the solution for motion of the spherical membrane must blow up in finite time and the life span of the solution is $\left[0, \bar{T}_{\max }\right)$, where $\bar{T}_{\max }$ is defined by (32) and $\bar{T}_{\max }<\infty$. (ii) When $r_{1}=v_{c}$, the solution also blows up in finite time and the life span of the solution is $\left[0, T_{\max }\right)$, where $T_{\max }$ is given by (28) and $T_{\max }<\infty$. (iii) When $r_{1}<v_{c}$, the solution exists globally on the time $t \in[0, \infty)$, and the solution satisfies the following decay conditions:

$$
r(t) \longrightarrow r_{+}, \quad r_{t}(t) \longrightarrow 0, \quad r_{t t}(t) \longrightarrow 0,
$$

when $t \rightarrow \infty$.

In geometry, our results show that, for a given spherical membrane centered at the black hole $r=0$, (i) if the initial velocity is larger than the critical velocity $v_{c}$, then singularities in the motion will develop in finite time, precisely, at the time $\bar{T}_{\max }$; that is, the spherical membrane disappears at the time $\bar{T}_{\max }$ in this case. (ii) If the initial velocity is equal to the critical velocity $v_{c}$, similar to the above case, singularities will develop in finite time, and the spherical membrane disappears at the time $T_{\max }$ in this situation. (iii) If the initial velocity is less than the critical velocity $v_{c}$, then motion of the spherical membrane will not stop at any finite time, and the spherical membrane converges to the event horizon $r=r_{+}$ when the time tends to infinity. From the graphs we see that motion of the spherical membrane may be different due to the different initial velocity. In this case, the moving velocity of the membrane goes to zero when the membrane tends to the event horizon.

\section{Acknowledgments}

This work was supported in part by the NNSF of China (Grant no. 11271323) and the Qiu-Shi Professor Fellowship from Zhejiang University, China.

\section{References}

[1] T. H. Colding and W. P. Minicozzi, II, Minimal Surfaces, vol. 4 of Courant Lecture Notes in Mathematics, New York University Courant Institute of Mathematical Sciences, New York, NY, USA, 1999.
[2] R. Osserman, A Survey of Minimal Surfaces, Dover, New York, NY, USA, 2nd edition, 1986.

[3] S. Brendle, "Hypersurfaces in Minkowski space with vanishing mean curvature," Communications on Pure and Applied Mathematics, vol. 55, no. 10, pp. 1249-1279, 2002.

[4] C. H. Gu, "A global study of extremal surfaces in 3-dimensional Minkowski space," in Differential Geometry and Differential Equations (Shanghai, 1985), vol. 1255 of Lecture Notes in Mathematics, pp. 26-33, Springer, Berlin, Germany, 1987.

[5] C. H. Gu, "A class of boundary problems for extremal surfaces of mixed type in Minkowski 3-space," Journal für die Reine und Angewandte Mathematik, vol. 385, pp. 195-202, 1988.

[6] H. Lindblad, "A remark on global existence for small initial data of the minimal surface equation in Minkowskian space time," Proceedings of the American Mathematical Society, vol. 132, no. 4, pp. 1095-1102, 2004.

[7] Y. Nambu, "Strings, monopoles, and gauge fields," Physical Review D, vol. 10, no. 12, pp. 4262-4268, 1974.

[8] B. M. Barbashov and V. V. Nesterenko, Introduction to the Relativistic String Theory, World Scientific, Teaneck, NJ, USA, 1990.

[9] M. Bordemann and J. Hoppe, "The dynamics of relativistic membranes. Reduction to 2-dimensional fluid dynamics," Physics Letters B, vol. 317, no. 3, pp. 315-320, 1993.

[10] M. Bordemann and J. Hoppe, "The dynamics of relativistic membranes. II. Nonlinear waves and covariantly reduced membrane equations," Physics Letters B, vol. 325, no. 3-4, pp. 359-365, 1994.

[11] J. Hoppe, "Some classical solutions of relativistic membrane equations in 4-space-time dimensions," Physics Letters B, vol. 329, no. 1, pp. 10-14, 1994.

[12] C.-L. He and D.-X. Kong, "Spherical symmetric solutions for the motion of relativistic membranes in the Schwarzschild spacetime," Journal of Mathematical Physics, vol. 50, no. 8, Article ID 083516, 15 pages, 2009.

[13] S.-J. Huang and D.-X. Kong, "Equations for the motion of relativistic torus in the Minkowski space $\mathbb{R}^{1+n}$," Journal of Mathematical Physics, vol. 48, no. 8, Article ID 083510, 15 pages, 2007.

[14] D. X. Kong and Q. Zhang, "Solution formula and timeperiodicity for the motion of relativistic strings in the Minkowski space $\mathbb{R}^{1+n}$, Physica D, vol. 238, no. 9-10, pp. 902922, 2009.

[15] D. X. Kong, Q. Zhang, and Q. Zhou, "The dynamics of relativistic strings moving in the Minkowski space $\mathbb{R}^{1+n}$," Communications in Mathematical Physics, vol. 269, no. 1, pp. 153-174, 2007.

[16] N. Xie, "An absence theorem for static wave maps in the Schwarzschild-AdS spacetime," Physics Letters A, vol. 338, no. 3-5, pp. 297-302, 2005. 


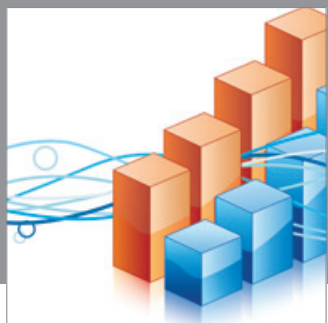

Advances in

Operations Research

mansans

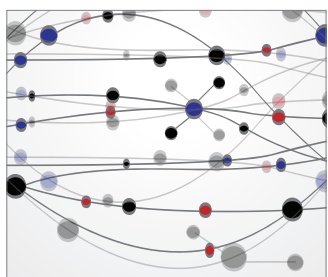

The Scientific World Journal
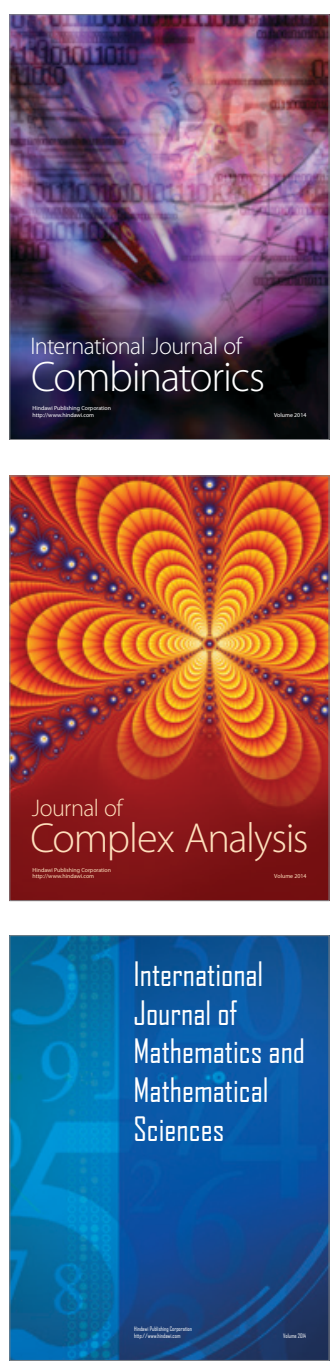
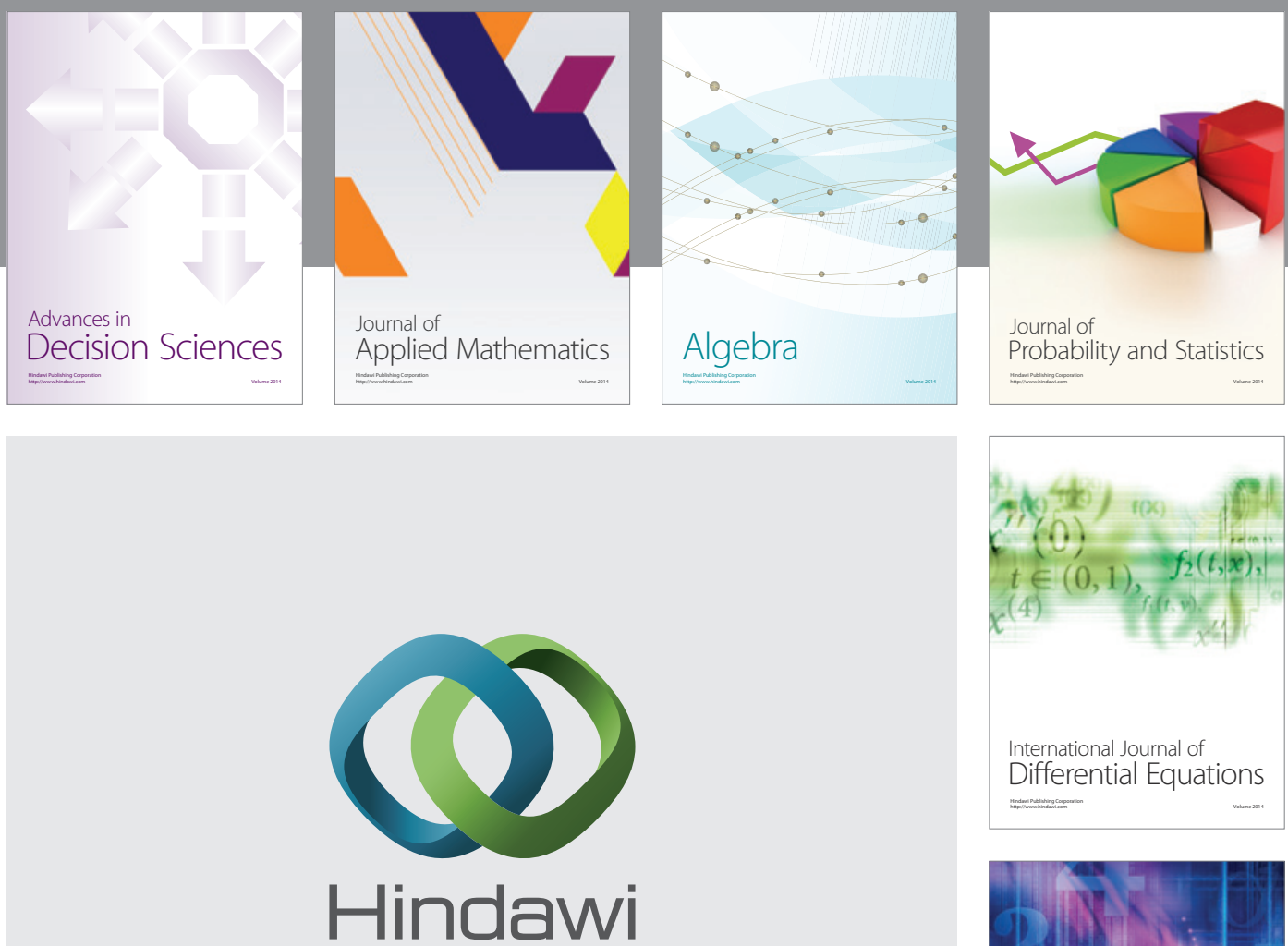

Submit your manuscripts at http://www.hindawi.com
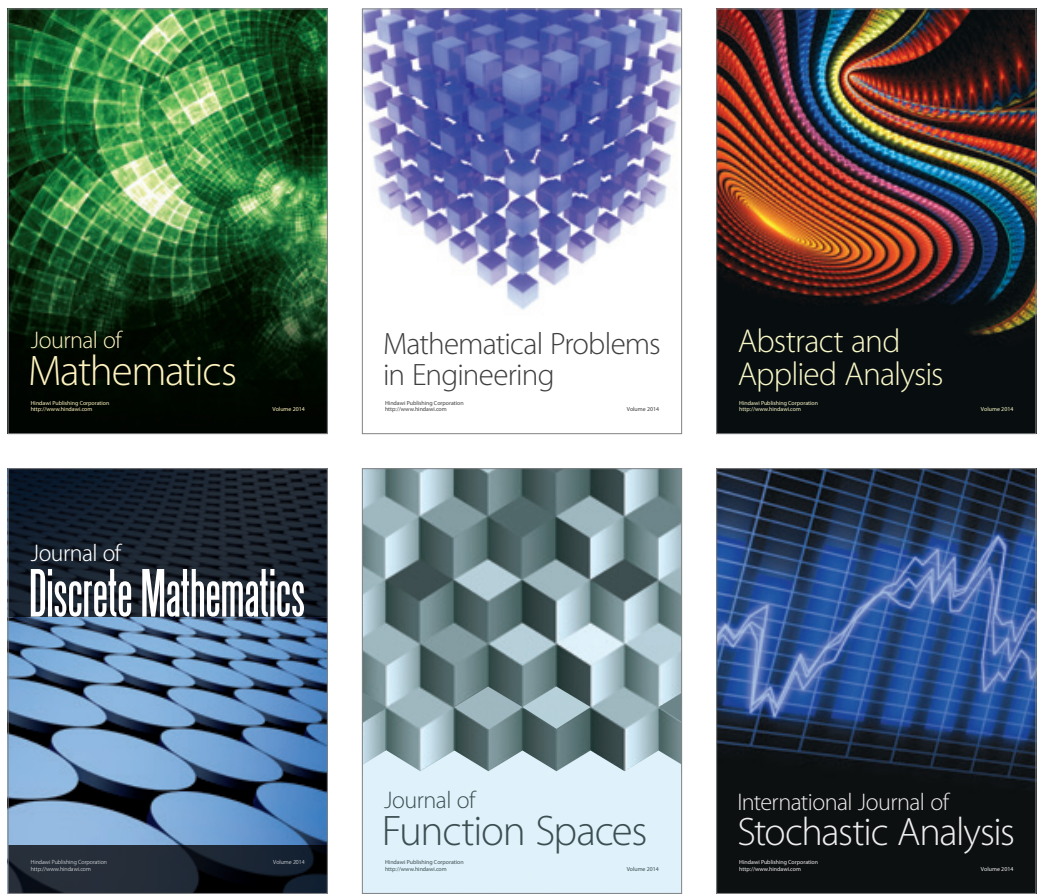

Journal of

Function Spaces

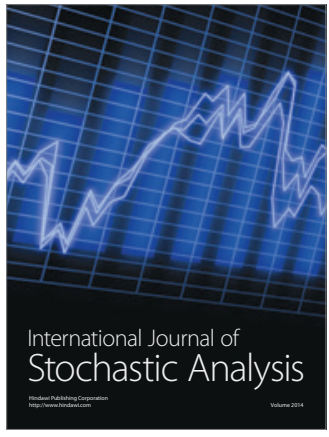

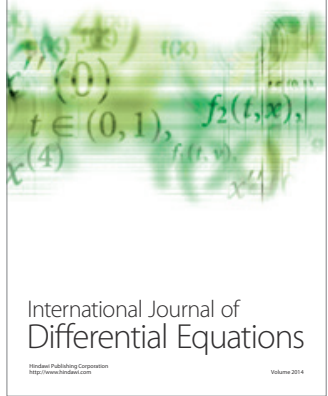
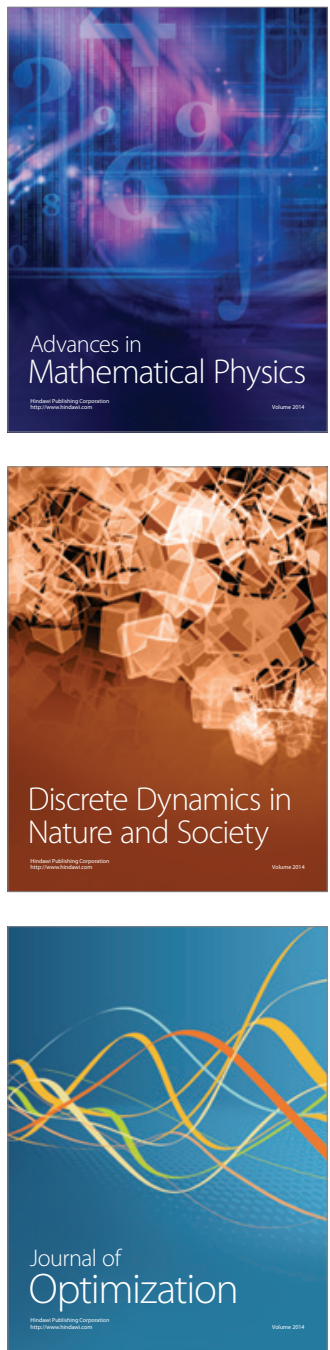